\title{
Treatment escalation plans: a review of patient and family discussions and communication between healthcare professionals
}

\author{
Authors: Catrin Manon Lewis, Eliza Lefroy and Billy Cheung*
}

\section{Introduction}

Treatment escalation planning (TEP) forms an integral part of patient management plans during an acute medical take. However, the quantity and quality of documentation can be variable. We analysed the documentation of components related to discussions with patients and families on our electronic TEP forms, identifying areas for improvement. We also looked at whether decisions were approved by responsible consultants and conveyed to nursing staff in a timely manner.

\section{Materials and methods}

Treatment escalation plans (TEPs) from four medical wards were reviewed. We looked at the number of forms discussed with patients and/or relatives. If there was no documented discussion, a reason was expected to be given. We also reviewed the number of forms approved by the responsible consultant within 24 hours, and the number of forms countersigned by the senior nurse in charge.

Two rounds of interventions were performed, with further data collections after each.

Following initial data collection on a single day in January 2018, an email from the medical director was sent to the medical consultant body to remind of the requirement to document discussions with patients and/or relatives.

A second review of forms was completed on another day in February 2018 following intervention.

The third data collection occurred in February 2019 following the presentation of our findings to the hospital-wide medical grand round, to highlight our previous findings.

\section{Results and discussion}

A total of 34 TEP forms were reviewed in Jan 2018. Of these, $23 / 34$ $(67.6 \%)$ were discussed with patients, with $16 / 34$ (47.1\%) discussed with families. 12/34 (35.3\%) were discussed with both.

41 forms were reviewed after the first intervention.

$21 / 41(51.2 \%)$ of TEP forms were discussed with the patients, with $23 / 41(56.1 \%)$ discussed with families and 10/41 (24.4\%) with both. Of forms not discussed with patients or families, $6 / 7$ had a documented reason, this improved to 7/7 in February 2018.

Authors: Homerton University Hospital NHS Foundation Trust ${ }^{*} \mathrm{RCP}$ chief registrar
Commonly documented reasons include patient lacking capacity or no family available for discussion, by phone or in person.

23/34 (67.6\%) of TEP forms were countersigned by a consultant within 24 hours but this dropped to 25/41 (60.9\%) in February 2018. This improved to $13 / 16$ (81.3\%) by February 2019 after grand round intervention.

The senior nurse in charge countersigned in less than $5 \%$ of cases in the first and second data collection rounds.

49 TEP forms were analysed in our third data collection.

$22 / 49(44.9 \%)$ were discussed with patients and 24/49 (49.0\%) were discussed with families and $15 / 49(30.6 \%)$ with both.

\section{Conclusion}

The documentation of TEP form discussions with patients and families remains inconsistent despite two different interventions. This may be explained by the rotation of junior doctors. We must therefore continue to highlight the TEP form requirements to each new cohort. Countersignature by the responsible consultant within 24 hours showed a more promising improvement. Further multidisciplinary training is required to improve rates of overall TEP completion, as well as ensuring all sections are fully completed. 